\title{
MEDIRAD formulation of science-based recommendations for medical radiation protection: a stakeholder forum survey
}

\author{
M. Benderitter ${ }^{1, *}$, E. Herrera Reyes ${ }^{1}\left(\mathbb{D}\right.$, M.A. Benadjaoud $^{1}$, F. Vanhavere $^{2} \mathbb{D}$, N. Impens ${ }^{2}(\mathbb{D}$, \\ U. Mayerhofer-Sebera ${ }^{3}$, M. Hierath ${ }^{3}$, J.R. Jourdain ${ }^{1} \oplus$, G. Frija ${ }^{4}$ and J. Repussard ${ }^{5}$ \\ ${ }^{1}$ Institut de Radioprotection et de Sûreté Nucléaire (IRSN), Pôle Santé, 31 avenue de la Division Leclerc, BP17, 92262 Fontenay-aux- \\ Roses, France. \\ 2 Belgian Nuclear Research Centre (SCK CEN), Boeretang 200, 2400 Mol, Belgium. \\ ${ }^{3}$ European Institute for Biomedical Imaging Research (EIBIR), Am Gestade 1, 1010 Vienna, Austria. \\ ${ }^{4}$ Université de Paris (UP), 85 Bd Saint-Germain, 75006 Paris, France. \\ 5 Jacques Repussard Conseil, 7 Allée de la Forêt de Marly, 78860 Saint-Nom-la-Bretèche, France.
}

Received: 19 August 2021 / Accepted: 12 October 2021

\begin{abstract}
MEDIRAD (Implications of Medical Low Dose Radiation Exposure) is an innovative European research project funded by EURATOM which seeks to bring closer together the nuclear and medical research communities in order to advance science for radiation protection in radiotherapy, nuclear medicine, and diagnostic and interventional radiology. The project also aims to promote links between science and society, with the goal of better protecting patients and professionals, through the publication of recommendations based on MEDIRAD research findings (http://www.medirad-project.eu/). The MEDIRAD Stakeholder Forum(SF) was designed to set up a dialogue between the Consortium member organisations and the society regarding the recommendations, which are expected from this project. We envisage three successive steps in this dialogue (1: first SF consultation identifying the needs for improved medical radiological protection; 2: drafting science based MEDIRAD recommendation and 3: second SF consultation to collect feedback), which are implemented throughout the project. A first overview of input of the Stakeholder Forum about the topics to be addressed in the MEDIRAD recommendations, based on an exploratory questionnaire, is presented in this article. Quantitative and qualitative in-depth analysis leads to the identification of 11 priority thematics.
\end{abstract}

Keywords: medical radiation protection / stakeholder / questionnaire / recommendation / MEDIRAD

\section{Introduction}

The evolution of medical science and the growing pace of innovation and deployment of medical technology have led to a situation where most artificial ionising radiation(IR) exposure of the European population is created by diagnostic imaging, nuclear medicine, and radiotherapy (RT) procedures. Medical radiation is an essential tool both in diagnosis and treatment. The increasing use of new modalities for diagnosis and treatment raises several issues regarding the radiological protection of patients and of medical workers. In Europe, thecontribution to the total population dose of CT, conventional radiography, fluoroscopy, interventional radiology and nuclear medicine procedures is respectively $57 \%, 17 \%, 12 \%$, 9\% and 5\% (BfS - Dose Datamed 2). Over 45 million CT scans

*Corresponding author: marc . benderitter@irsn.fr are delivered annually in Europe (EC - Eurostat $)$ with an average yearly increase of around $6 \%$ in recent years. In addition, 1.6 million RT treatments are performed annually across the EU (Rosenblatt et al., 2013), which also cause some exposure to healthy tissues. Based on projected cancer distributions, a $16 \%$ expected increase in the number of RT treatment courses is estimated by 2025 in Europe (varying between countries from less than 5\% to more than 30\%) (Borras et al., 2016).

The rise in the number of medical examinations using ionising radiation, such as CT scans, has led to an increased collective dose for patients at the European level, with a potentially heightened risk for patients undergoing repeated procedures, especially for young patients (Bernier et al., 2018; Masjedi et al., 2019). There is a need to evaluate the health effects of these exposures, optimise practices to reduce doses, and develop dose evaluation tools that can be used in clinical practice to ensure adequate and improved radiation protection for patients and medical professionals. However, these risks 
are currently not well quantified. Moreover, although latent risks to healthy tissues resulting from radiotherapy, interventional radiology and nuclear medicine protocols have significantly been reduced with modern technologies (Tsapaki, 2020), these risks remain an important issue as the rate of longterm patient survival increases (Skinner et al., 2006). Finally, radiation protection for medical professionals with potentially high levels of exposure can still be further improved.

Further radiation protection progress is bound to continue through technological innovation, good professional practice, and more individualised patient care. This progress depends on continually accumulating scientific knowledge that can serve to address several public health challenges, and on ensuring a fast transfer of this new knowledge from research to practice.

Multidisciplinary (expertise and science, science and medicine) Europe-wide research can bring forward innovative approaches and foster solutions beyond the reach of single countries and research institutions for the benefit of millions of European patients and tens of thousands of medical professionals (EANM et al., 2017). Recent progress in biosciences and computational technologies (i.e., "Big Data" and artificial intelligence) (Hosny et al., 2018; Francolini et al., 2020) will help to reach some research goals. However, an effective linkage between science and society remains essential to drive research priorities, set appropriate research guidelines (Turcanu et al., 2020) and ethics rules (Meskens, 2020), and accelerate the transition from research results to daily medical practice; thus providing individual patient access to optimised diagnostic and therapeutic protocols.

Instruments for this linkage process include:

- European and national research policymaking;

- Regulatory environment-both for research and implementation of the radiation protection aspects of the Basic Safety Standards reflecting an international consensus on what constitutes a high level of safety for protecting individual and the environment from harmful effects of ionising radiation (IAEA, 2014, 2018);

- Diagnostic reference levels (DRL) which have proven to be an effective tool that aids in optimisation of protection in the medical exposure of patients for diagnostic and interventional procedures (ICRP, 2017);

- Science-based professional good practice guidelines;

- Technology standardisation;

- Patient information and patient dialogue with medical professionals (IAEA, 2014, 2018).

In this context, MEDIRAD is an innovative European research project funded by EURATOM which seeks to advance science for radiation protection in a selected areas in medicine, through four scientific "Work Packages (WP)", dedicated to the following aspects: WP-2 "Dose evaluation and optimisation in medical imaging"; WP-3 "Impact of low dose radiation exposure from I-131 radioiodine ablation of thyroid cancer"; WP-4 "Breast radiotherapy and secondary cardiovascular risks: establishing risk models for clinical support" and WP-5 "Possible health impact of paediatric scanning-a molecular epidemiology study". MEDIRAD aims to promote links between science and society, with the view of better protecting patients and professionals, through the publication of recommendations based on MEDIRAD research findings, and taking into account the priority expectations of user's. Traditionally, the periodic updating of regulations plays a major role in the process of enhancing radiation protection practice. This process is largely based on a lengthy analysis of scientific findings, mostly carried out by a limited number of experts within UNSCEAR and ICRP. This regulatory approach is not based on the development of a bottom-up strategy of professional consensus, with an active collaboration with endusers, and regular stakeholder consultations tend to occur at a late stage of development of regulations. This may have some negative repercussions on the practical implementation of regulatory measures. The non-use of clinical imaging referral guidelines, though defined as mandatory in Directive 97/43 and 2013/59 for medical imaging, or the lack of harmony across Europe of professional practice in the field of DRL's illustrate the limitations of this approach. MEDIRAD utilises a novel procedure, through a consensus building approach for the development of recommendations, involving a large panel of stakeholders, including medical professionals and representatives of policy bodies and allowing direct exchanges between the different communities, which may accelerate and facilitate the uptake of research results towards enhancing medical radiation protection.

The implementation and development of the MEDIRAD stakeholder related activities rely on a two-level structure: a Stakeholder Board (SHB) and a Stakeholder Forum (SF). To engage in a meaningful dialogue with stakeholders, it was foreseen that a MEDIRAD SF would be set up. 186 organisations were invited to join the Stakeholder Forum. The SHB consists of eleven experts bringing together one representative of each of European platforms and medical societies involved in medical radiation, and international organisations. The SHB also includes a patient representative identified through the mechanisms of the European Patients' Forum and the ESR Patient Advisory Group. According to the MEDIRAD Grant Agreement, the SHB (i) supports the coordination of the MEDIRAD stakeholder related activities, (ii) formulates proposals on the composition of the SF, (iii) advises on the design and content of the web-based stakeholder consultation, (iv) contributes in an advisory role to the development of the MEDIRAD recommendations, (v) gives views and thoughts on the most suitable ways to ensuring appropriate promotion and dissemination of the MEDIRAD outcomes to concerned stakeholders.

The MEDIRAD SHB proposed to carry out a broad ranging exploration of stakeholder expectations through a distribution of an "exploratory questionnaire", across Europe and beyond. This article describes the MEDIRAD SF exploratory questionnaire and depth analysis of the findings.

\section{Methodology}

\subsection{Implementation of the SHB and SHF}

The following representatives have been appointed as members of the MEDIRAD SHB the following organisations: EPF (European Patients Forum) and EANM (European Association of Nuclear Medicine), EFOMP (European Federation of Organisations for Medical Physics), EFRS (European Federation of Radiographer Societies), ESR (European Society of Radiology), ESTRO (European Society 
for Radiotherapy and Oncology), EURADOS (European Radiation Dosimetry Group), HERCA (Heads of the European Radiological Protection Competent Authorities), ICRP (International Commission on Radiological Protection), MELODI (Multidisciplinary European Low Dose Initiative), and WHO (World Health Organization) as a special advisor. To engage in a meaningful dialogue with stakeholders, it was foreseen that a MEDIRAD SF would be set up. This SF with up to 150 members will interact with the project exclusively through a dedicated web-based platform. From the 186 stakeholder invited organisations, 86 confirmations, from across $22 \mathrm{EU}$ member states, were received, and currently constitute the SF membership. In total, $85 \mathrm{SF}$ members responded to the questionnaire. Through this stakeholder dialogue process, members of SHB/SHF were able to observe ongoing research activities, and access documents from MEDIRAD. In order to ensure that this consultation process is, as a whole, ethically correct and may as such contribute to the future publicly available results of MEDIRAD, SHB and SF members comply with the following three principles: disclosure of interests, confidentiality and transparency.

\subsection{Elaboration of the SF questionnaire}

A questionnaire directed to SF participants was prepared to collect their concerns and expectations related to the MEDIRAD recommendations towards better enhancing the radiation protection of the public, workers and patients. This questionnaire included a statement highlighting that the SF members' contributions would not be interpreted as an endorsement by responders of MEDIRAD recommendations. The questionnaire was drafted based on the R\&D objectives of MEDIRAD, taking into account the results of a meeting between WP leaders and MEDIRAD SHB members to discuss the plans for the elaboration of the recommendations. The draft questionnaire, including its introductory text, was then submitted for comments to the SHB members, to MEDIRAD WP leaders and the MEDIRAD scientific and clinical coordinators. The questionnaire consisted of several sections: (i) a first set of questions was about the ethics of the MEDIRAD SF, including ethical issues related to SF members' contributions to the elaboration of MEDIRAD recommendations; (ii) a second set of questions concerned the ranking of broad-ranging approaches with the goal of optimising the exposure to IR of patients and medical professionals; (iii) there are several complementary ways for reducing and optimising ionising radiation exposure for patients and medical staff. Science evidence-based approach exerts a significant influence over time, on the evolution of techniques, medical practices, as well as regulatory oversight methods. A third set of questions proposed a selection of 25 technical topics, to be ranked and commented, to consider their possible inclusion in the MEDIRAD recommendations.

\subsection{In-depth analysis of the SF questionnaire}

Quantitative and semi-qualitative analysis of the SF questionnaire was performed. The answers were jointly analysed according to their priority contingency tables. A global score was defined for each technical topic as a weighted sum of each priority frequency (weights of $-2,-1,1$ and 2 for the low interest, low priority, and intermediate and high priority response, respectively). A cluster analysis (Kmeans algorithm) based on these global scores was conducted to identify the main response features. The optimal number of clusters was determined using the silhouette criteria, which measures how close each response in one cluster is to responses in the neighbouring clusters (Kaufman and Rousueeuw, 1990). In order to investigate the structural organisation of the different technical topics, a principal nonlinear component analysis specially designed for categorical data (PRINCALS GIFI framework (Gifi, 1990) was conducted to extract the coordinates of the vertices corresponding to each technical topic, in a Euclidean plan. These coordinates (also called component scores) are then used to display a scatterplot: each technical topic is represented as an individual point. This approach ensures that two technical topics with similar scoring patterns will be represented by closely located points.

The main statement associated to each question was shortened to facilitate their representation. For example: (i) "Improved protocols aimed at reducing exposure whilst preserving or improving diagnostic quality/therapeutic benefits" to "Protocol to reducing exposures"; (ii) "Increasing education and training of medical professionals on radiation protection optimisation" to "E\&T". The semi-qualitative analysis was possible based on the proposal for each topic for priority evaluation by the SF. Open comments were possible for each question proposed to the SF. The respondents were invited to comment with their own words and 5 to 10 comments were provided by SF members for each question. The semi-qualitative analysis was conducted by extracting the keywords from the comments that were grouped by technical topics. Dendrograms were elaborated to drive options for the recommendation. Semiquantitative analysis was also proposed by identifying qualifying adjectives (important, challenging, useful or others), which the respondents frequently used for each topic. A percentage of the positive or negative evaluation was assessed to quantify the degree of consensus among SF members on the proposed qualifying adjective. The most frequently cited qualifying adjective was reported in the figures.

\section{Results and discussion}

\subsection{European stakeholders answering to the questionnaire}

From the 186 stakeholder invited organisations, 86 confirmations were received, and currently constitute the SF membership. In total, $85 \mathrm{SF}$ members responded to the questionnaire. Among them were 69 national stakeholders from Europe (Fig. 1) and the rest, from other regions including North America, Asia Middle East and also from international organisations.

From the total number of respondents, a predominant number of medical physicists $(41 \%)$ replied to the questionnaire followed by medical doctors $(31 \%)$, mostly radiologists (Fig. 1). It is noticeable that epidemiologists $(7 \%)$ and biologists $(1 \%)$ did not provide so many answers. These considerations are important to highlight when the results of the questionnaire are analysed. 

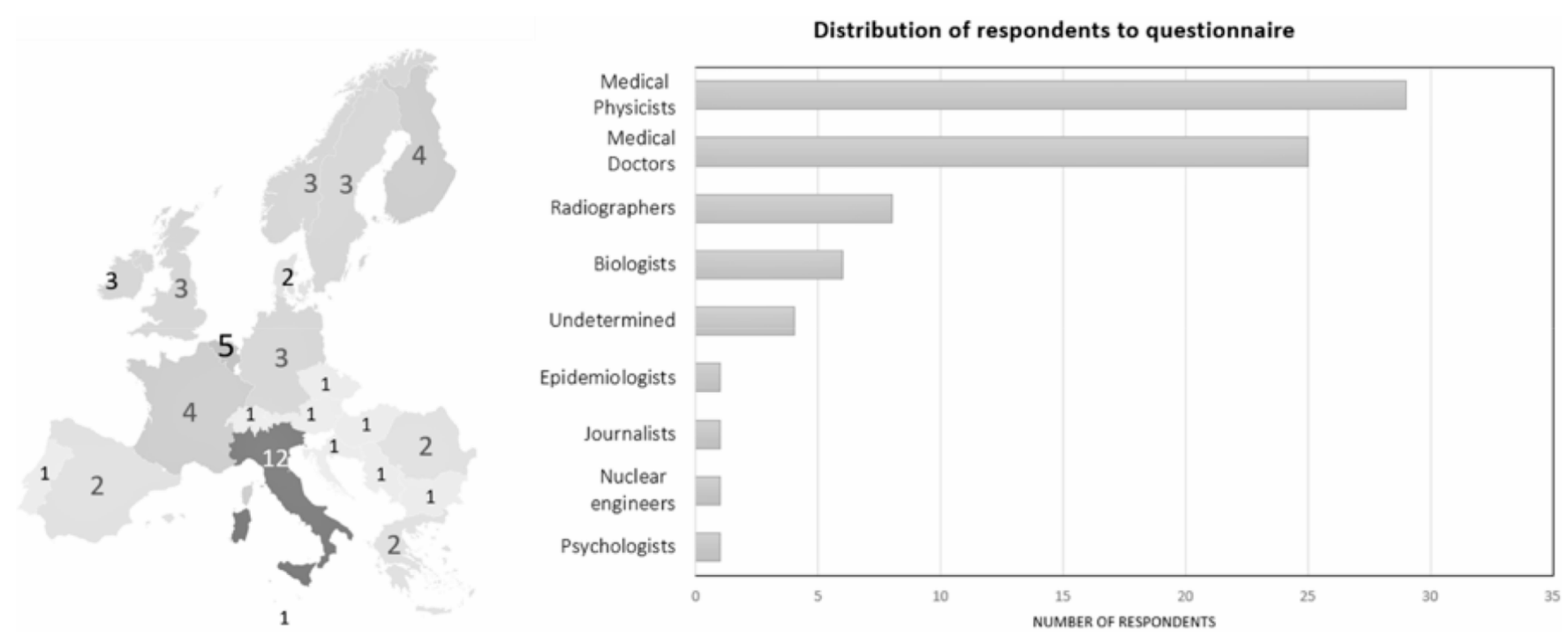

Fig. 1. European stakeholders answering to the questionnaire. Left: Number of national stakeholders confirmed and its distribution in Europe. Right: Distribution of respondents to the questionnaire according to their professions, based on public information available on Internet.

Table 1. European stakeholders' expectations: High Priority technical topics.

\begin{tabular}{ll}
\hline Rank & Topics \\
\hline 1 & Optimising image quality/dose during CT scans, including multimodality imaging procedures (e.g., SPECT-CT and PET-CT-scans). \\
2 & $\begin{array}{l}\text { Improved protocols aimed at reducing exposure whilst preserving or improving diagnostic quality/therapeutic benefits (e.g., better } \\
\text { accounting of potential secondary or late effects of healthy tissue exposure). }\end{array}$ \\
3 & $\begin{array}{l}\text { Optimising patient follow-up care after radiation therapy and collecting valuable epidemiological data through a better linkage } \\
\text { of medical professionals from relevant disciplines. }\end{array}$ \\
Increasing education and training of medical professionals on radiation protection optimisation.
\end{tabular}

\subsection{Quantitative analysis}

The clustering analysis concluded to an optimal partitioning of the 25 technical topics in four groups shown in Tables 1-4. By analysing the 25 technical topics response dynamic in Figure 2, these clusters can easily be interpreted in term of priority importance as illustrated by the following examples: (i) High priority technical topics: those with the highest frequency of replies and scores with an "exponential growing" dynamics (Fig. 2a: black bars).

For example, the variables "Optimising image quality/dose during CT scans" (Rank 1) or "Optimising patient follow-up care after radiation therapy and collecting valuable epidemiological data" (Rank 3 ) have a similar score represented by the black lines in the graphs in Figure $2 \mathrm{~b}$ and could be classified as "high" priority technical topics. (ii) Low interest technical topics: those with the lowest frequency of replies and scores with an "inverted U" dynamics shaped (Fig. 2b: dotted lines). For example, the variables "Web smartphone application for adverse effects" (Rank 25) or "Reinforcing regulation" (Rank 24) have a similar score represented by dotted lines in the graphs in Figure 2b and were classified by the SF as "low interest" technical topics. (iii) Intermediate/low priority technical topics: It comprises respectively the dark and light grey bars (Fig. 2a) technical topic groups which exhibit a strictly monotone variation with respectively an increasing plateau (dark grey lines in Fig. 2c) and a stable plateau (light grey lines in Fig. 2c). For example, "Promoting individualised patient care in nuclear medicine. Procedures for evaluating patientspecific doses deliver to volumes and organs through activity uptake" (Rank 5) and "Improvement of target definition by better delineation of the target volume, better margins definition and better definition of the heterogeneity and of the biological volumes of the tumour at the voxel scale" (Rank 6) have scoring dynamics positioned as intermediate priority technical topics (dark grey lines in Fig. 2c). In the same way, "Technology development" (Rank 12) and "Further radiation protection research for radiation oncology: normal tissue response" (Rank 13) have scoring dynamics positioned as low priority technical topics (light grey lines in Fig. 2c).

The scatterplot of the 25 technical topics based on the first two component scores (see. Methodology section) is represented in Figure 3. According to the first dimension, the 25 technical topics have been arranged in the same order than previously obtained using the global priority score. The technical topics designated as "highly" (black bars and lines in Fig. 2) and "intermediate" (dark grey bars and lines in Fig. 2) are located in the right side of the plot (Fig. 3). On left side, "low interest" (white bars and dotted lines in Fig. 2) and "low priority" (light grey bars and lines in Fig. 2) are observable.

Such representation also gives a measure of "proximity" of some technical topics with regard to the SF participant 
Table 2. European stakeholders' expectations: Intermediate Priority technical topics.

\begin{tabular}{ll}
\hline Rank & Topics \\
\hline 5 & $\begin{array}{l}\text { Promoting individualised patient care in nuclear medicine. Procedure for evaluating patient-specific doses deliver to volumes } \\
\text { and organs through activity uptake. }\end{array}$ \\
6 & $\begin{array}{l}\text { Improvement of target definition by better delineation of the target volume, better margins definition and better definition of the } \\
\text { heterogeneity and of the biological volumes of the tumour at the voxel scale. }\end{array}$ \\
7 & $\begin{array}{l}\text { Modelling of patient dosimetry at the voxel scale. It is necessary to move from planned dose maps to delivered dose maps. } \\
\text { (Treatment planning improvement, doses delivered during diagnostic and positioning imaging procedures, modelling simulations, } \\
\text { clinical Decision Support System, Data standardisation and machine learning data base...). }\end{array}$ \\
& $\begin{array}{l}\text { Predicting quickly and accurately the response of tumours and normal tissues to ionising radiation using new multimodal } \\
\text { and functional imaging and/or new biological and molecular surrogates. The development and validation of novel biomarkers }\end{array}$ \\
will be required in order to develop treatment personalisation approaches. & $\begin{array}{l}\text { Development of European registries of patient dose/imaging with recommended appropriate quantities (effective dose, organ dose) } \\
\text { for radiological examinations. }\end{array}$ \\
Developing and validating operational biomarkers predictive of patient exposure - side or late adverse effects - following repeated \\
radiological examinations, or radiotherapy protocols. \\
Optimising medical staff protection during interventional radiological procedures by ensuring proper availability and
\end{tabular}

Table 3. European stakeholders' expectations: Low Priority technical topics.

\begin{tabular}{ll}
\hline Rank & Topics \\
\hline 12 & Technology development. \\
13 & Future radiation protection research for radiation-oncology: Normal tissue response. \\
14 & Development of European patient registries of dose/image/clinical diagnosis and patient follow-up, for the purpose of clinical \\
& procedure standardisation and radiation protection optimisation (European radio-vigilance). \\
15 & Future radiation protection research for radiation-oncology: Combined treatment. \\
16 & Modelling of patient dosimetry on an individual basis by highlighting the range of absorbed doses delivered from \\
& fixed administrations of activity, in order to evaluate the range of possible secondary effects, including long-term risks of \\
17 & secondary malignancies. \\
\hline
\end{tabular}

Table 4. European stakeholders' expectations: Low Interest technical topics.

\begin{tabular}{ll}
\hline Rank & Topics \\
\hline 18 & $\begin{array}{l}\text { Facilitating the development of large-scale multinational epidemiological studies by proposing guidelines to help } \\
\text { European countries to implement at the national level European regulatory requirements on ethics (including compliance } \\
\text { with GDPR directive). } \\
\text { Development of personalised protocols that factor in individual patient radiation sensitivity (e.g., via biomarkers } \\
\text { of radiation sensitivity). } \\
\text { Exploring of the potential of patient-specific radiobiology tests to assess individual radio-sensitivity, in order } \\
\text { to personalise treatment protocols. } \\
20\end{array} \quad \begin{array}{l}\text { Protocols to set up optimised imaging systems for quantitative imaging of I-131 irrespective of camera make or model. } \\
\text { Outlining a plan for a large-scale and multi-site epidemiological study to evaluate the effects of low absorbed doses } \\
\text { of radiation as a result of nuclear medicine imaging procedures in a population with an expected normal life expectancy. } \\
\text { Consideration of individual bio-kinetics in patients with residual thyroid tissue or adjuvant disease, rather than reliance } \\
\text { on models and values established for a healthy population. } \\
\text { Reinforcing regulations (e.g., by extending the scope of Diagnostic Reference Levels (DRLs) at the European level), } \\
\text { and regulatory oversight (e.g., radiation protection experts, inspections). }\end{array}$ \\
Web/smartphone application for adverse effects.
\end{tabular}



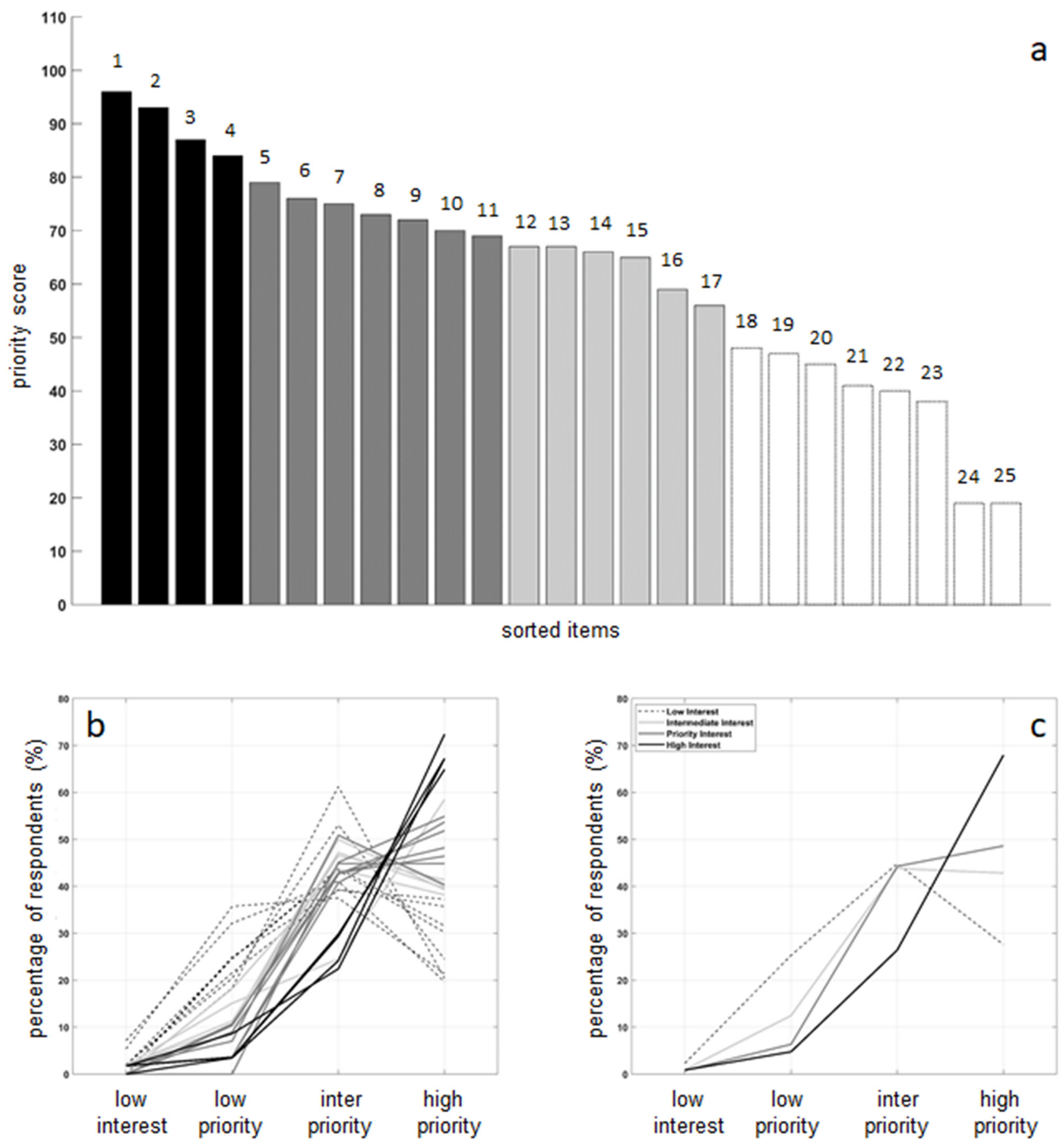

Fig. 2. Quantitative analysis. (a) Graph representing the 25 technical topics by priority scores: black bars: high priority; dark grey bars: intermediate priority; light grey bars: low priority technical topics; and white bars: low interest technical topics. (b and c) Twenty-five technical topics' profiles grouped according to the clustering based on the global priority: black lines: high priority; dark grey lines: intermediate priority; light grey lines: low priority technical topics; and dotted lines: low interest technical topics. (b) The graph clustering with the 25 technical topics and their pattern. (c) The technical topics analysed and classified by priority.

responses. For example, "improvement of target definition" and "modelling of patient dosimetry at the voxel scale" associated points appear close by, suggesting that these issues are often shared among the rating respondent. In contrast, this scatterplot highlights the remoteness of the "web/smartphone applications" and "reinforcing regulations" labels from the rest of the technical topics. In order to investigate the robustness of the technical topics clustering above, a sensitivity analysis was conducted by stratifying according to the main participant profession (Medical physicist, medical doctor, radiographer and radiobiologist) (data not shown). It concluded that the major "Top 11" technical topics obtained by pooling the "high" 


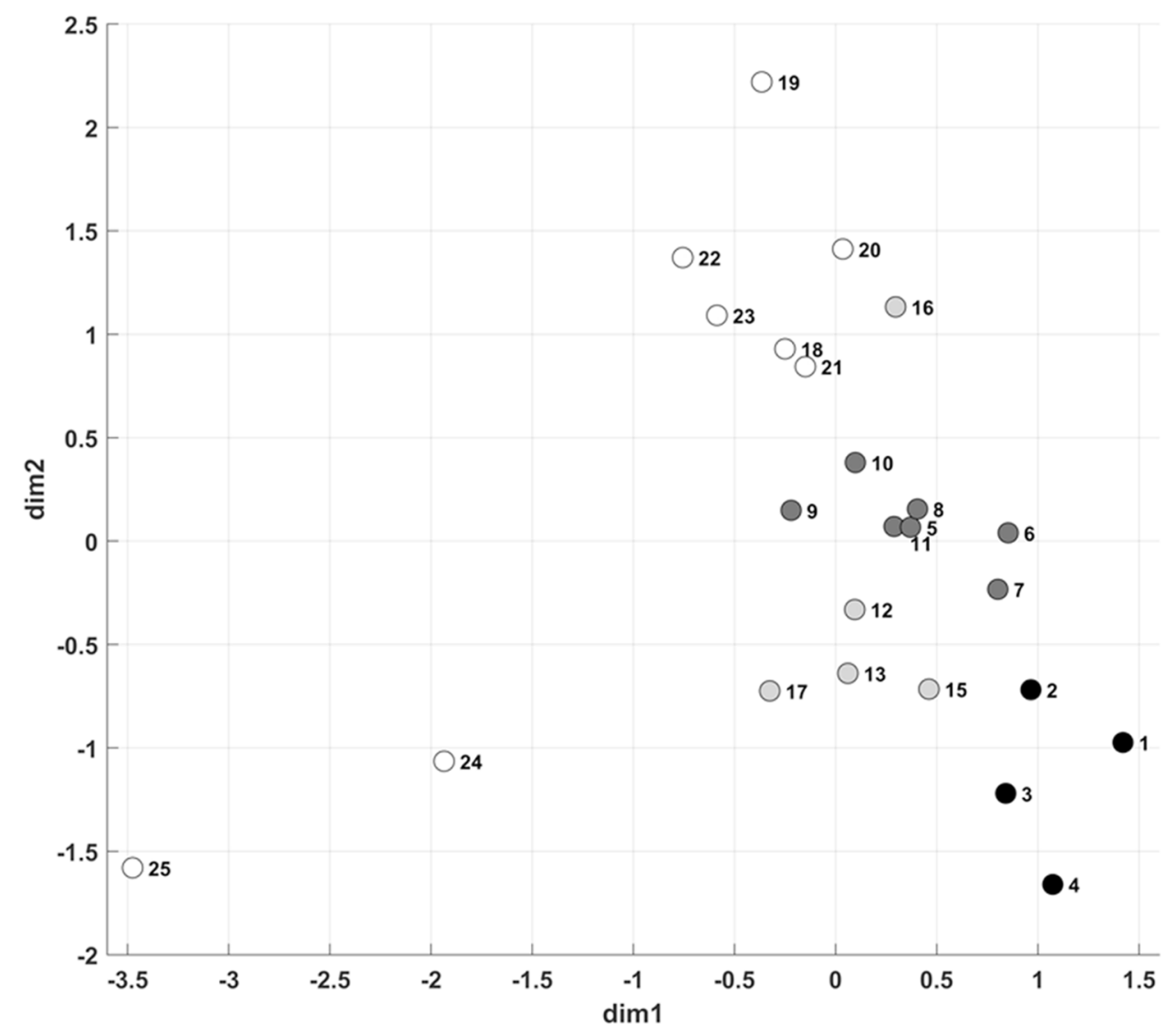

Fig. 3. Quantitative analysis: Two-dimensional reduction Scatterplot of the SF questionnaire technical topics. The four clusters previously identified in Figure 2a are represented with the same colour: black: high priority; dark grey: intermediate priority; light grey: low priority; and white: low interest.

and "intermediate" technical topic clusters (in black and grey in Figs. 3 and 4) were globally conserved across the professional strata.

In order to investigate the robustness of the 25 technical topics clustering above, a sensitivity analysis was conducted by stratifying according to the two main participant professions responding to the questionnaire (medical doctor and medical physicist). It concluded that the major "Top 11" technical topics obtained by pooling the "high" and "intermediate" priority were globally conserved across the professional strata.

\subsection{Qualitative analysis}

Current research performed within the MEDIRAD consortium is about the effects of medical ionising radiation in a variety of medical fields.

\subsubsection{Optimising image quality/dose during CT scans, including in multimodality imaging procedure (i.e., SPECT-CT and PET-CT scans)}

Among a list of three research questions that are currently being addressed by members of the MEDIRAD consortium in the field of Radiology and medical imaging, the SF puts high priority on "Optimising image quality/dose during CT scans, including in multimodality imaging procedure (i.e., SPECT-CT and PET-scans)". According to the SF, efforts for optimising image quality/dose are driven by clinical needs. The SF points out two main clinical needs including (i) diagnostic CT scan for paediatric application and (ii) diagnostic CT (PET and SPECT) for the target definition in radiation oncology. The SF suggests different reflection tracks to optimise dose delivery versus image quality during CT scans including normalisation of the test object to portray, or optimisation of the image quality but also image consistency. According to the SF's opinion, this objective requires high interaction with the industry. The semi-quantitative analysis of the provided comments confirmed the consensual position of the SF (71\%) on the "essential" nature of the proposed avenues towards further optimisation of exposures (Fig. 5).

\subsubsection{Promoting individualised patient care in nuclear medicine. Procedure for evaluating patient-specific doses deliver to volumes and organs through activity uptake}

In the field of Nuclear Medicine, among a list of 6 research questions that are currently being addressed by members of the MEDIRAD consortium, the SF puts priority on "Promoting individualised patient care in NM" and more specifically on "Procedure for evaluating patient-specific dose delivered to volume and organs through activity uptake". According to the $\mathrm{SF}$, efforts for promoting individualised patient care in NM are 
a

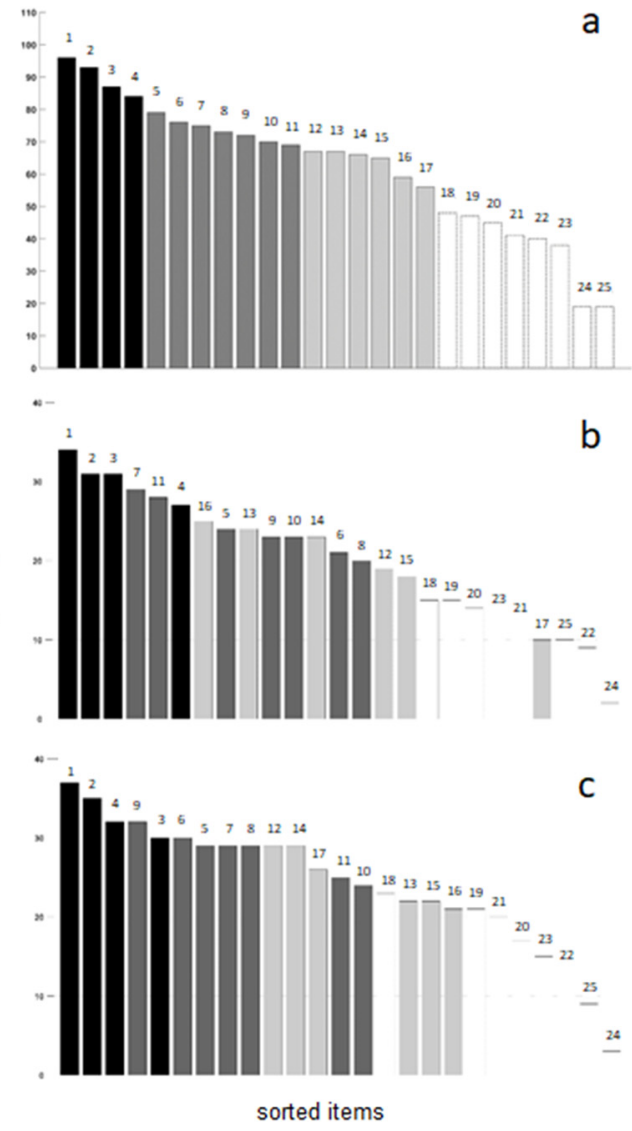

Fig. 4. Quantitative analysis: Stratified analysis of the global priority score according to participant profession. (a) Graph representing the 25 technical topics (listed in Tabs. 1-4) by priority scores: black bars: high priority; dark grey bars: intermediate priority; light grey bars: low priority technical topics; and white bars: low interest technical topics. (b) Graph representing the 25 technical topics by priority scores as analysed by the medical doctors. (c) Graph representing the 25 technical topics by priority scores as analysed by the medical physicist.

driven by clinical needs. Radionuclide uptake is patient dependent, and dosage according to general recommendations is inadequate. Therefore, the SF recommends a revision of the European council directive (Council Directive 2013/59 Euratom). This EU directive mandates that treatments should be planned so that exposures of target volumes shall be individually planned, and their delivery appropriately verified, as is the case for external beam radiotherapy. However, most of the current treatments still follow the historical "one size fits all" approach consisting of the administration of a nominal activity of the radiopharmaceutical. Thus, the personalised treatment planning issue must be addressed to provide an accurate and individualised knowledge of the radiation absorbed dose to the target and healthy critical tissues. One of the main reasons for a reluctance to perform individual patient dosimetry is that the process is complicated because there are currently no standard methods for calibrating or implementing radio-theranostic dosimetry in the clinic. Clinical dosimetry is a multi-step process. The key steps in obtaining accurate dose estimates are: (i) measurement of the activity to be administered, (ii) quantitative assessment of the spatial and temporal activity distribution in cells/tissues and (iii) calculation of the deposited energy from the activity distribution in the cells/tissues of interest. In case of high-LET radiation, the characteristics that make it attractive for targeted therapy also imply several challenges for the dosimetry of these new radiopharmaceuticals. The semi-quantitative analysis of the provided comments confirmed a consensual position of the SF (71\%), considering it as "necessary" to address this topic (Fig. 6).

\subsubsection{Technology development}

There are several complementary avenues for reducing and optimising ionising radiation exposure for patients (diagnostic and therapeutic) and medical staff. Research priorities and results tend to exert a significant influence over time, on the evolution of techniques, medical practices, as well as regulatory oversight methods. In this field, among a list of five research questions currently being addressed by members of the MEDIRAD consortium, the SF puts low priority on "technology development" for CT scan, radiotherapy technologies, vectorised radionuclides or innovative protective equipment. According to the SF, efforts for improved "Technology development" are a highly time and resourceconsuming effort that also requires approval and certification processes by national safety authorities. A market survey is of course mandatory (Efficacy, quality and performance of medical devices and radiation emitting products) before launching new innovative medical devices. According to the $\mathrm{SF}$, this objective requires high interaction with the industry. Technology improvement is in the hands of manufacturers. The semi-quantitative analysis of the provided comments confirmed a good consensus of the SF on the "importance" of this topic (75\%) (Fig. 7).

\subsubsection{Facilitating the development of large-scale} multinational epidemiological studies by proposing guidelines to help European countries to implement at the national level European regulatory requirements on ethics (including compliance with GDPR directive)

In the field of Radiobiology and Epidemiology, among a list of three research questions that currently being addressed by members of the MEDIRAD consortium, the SF puts low interest on "Facilitating the development of large scale multinational epidemiological studies by proposing guidelines to help European countries to implement at the national level European regulatory requirements on ethics (including compliance with GDPR directive)". The ultimate goal of this topic is a better accounting of potential acute and late effects of healthy tissue exposure. According to the SF, efforts for this topic require the set-up of EU platforms for data repository and the elaboration of technical guidelines to facilitate the development of large EU epidemiological studies. This objective seems difficult according to the SF to be reached. The SF also points out the need for grouping ethical commissions of the big EU research centre to facilitate compliance with the GDPR directive (Fig. 8). 


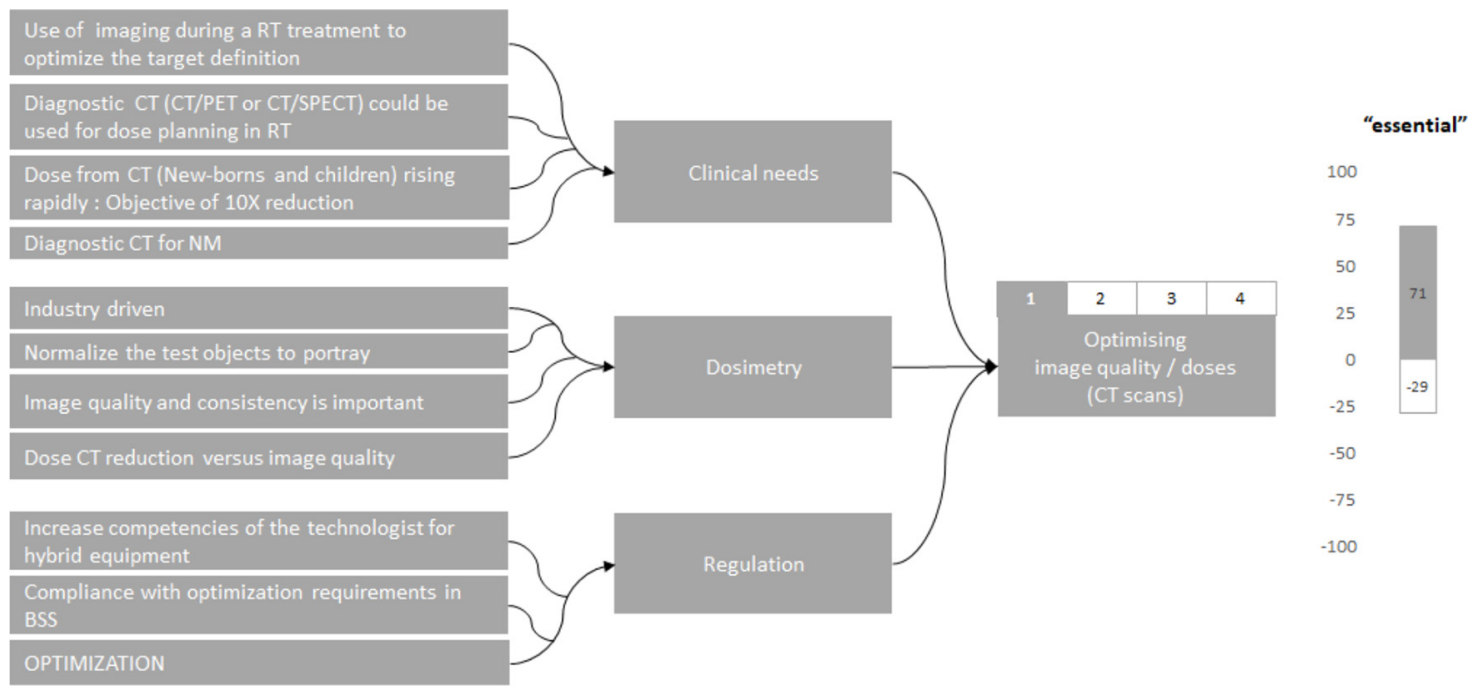

Fig. 5. Dendrogram for the high/medium MEDIRAD PRIORITY "Optimising image quality/CT dose" (rank 1/TOP11).

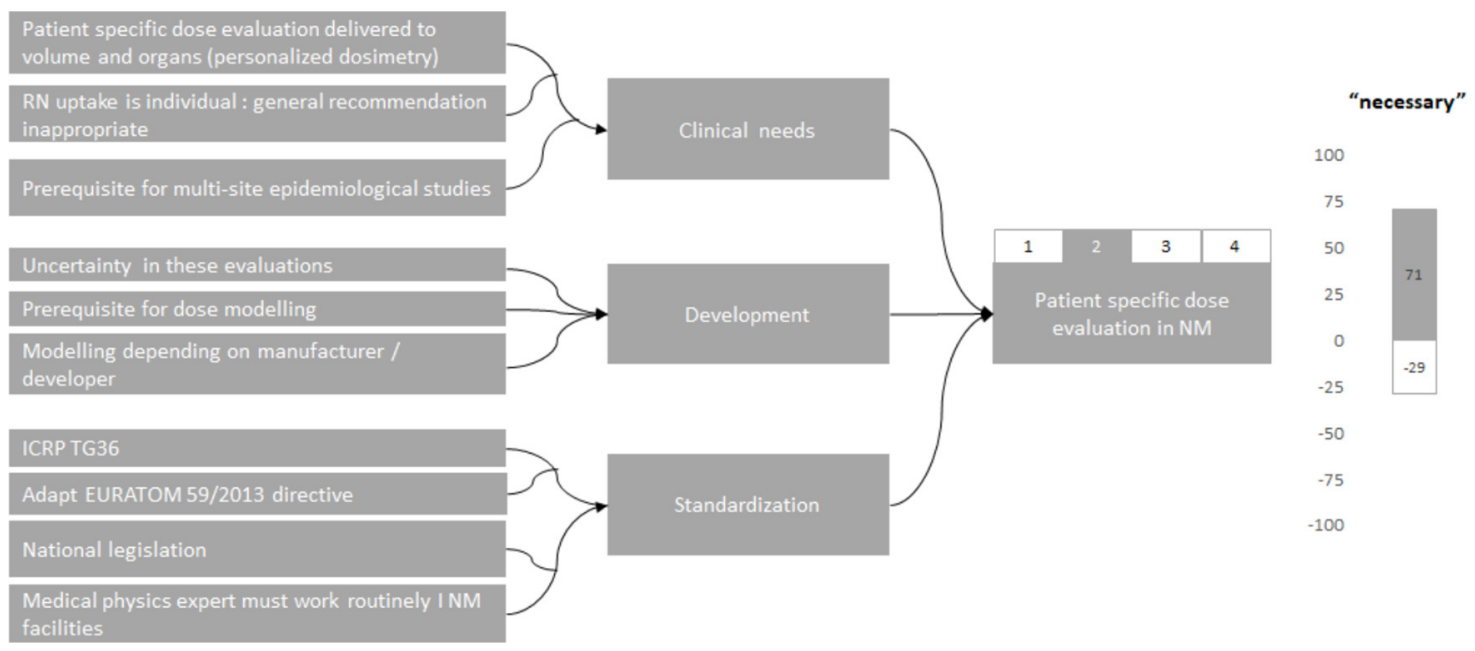

Fig. 6. Dendrogram for the high/medium MEDIRAD PRIORITY "Promoting individualised patient care in Nuclear Medicine" (rank 5/TOP11).

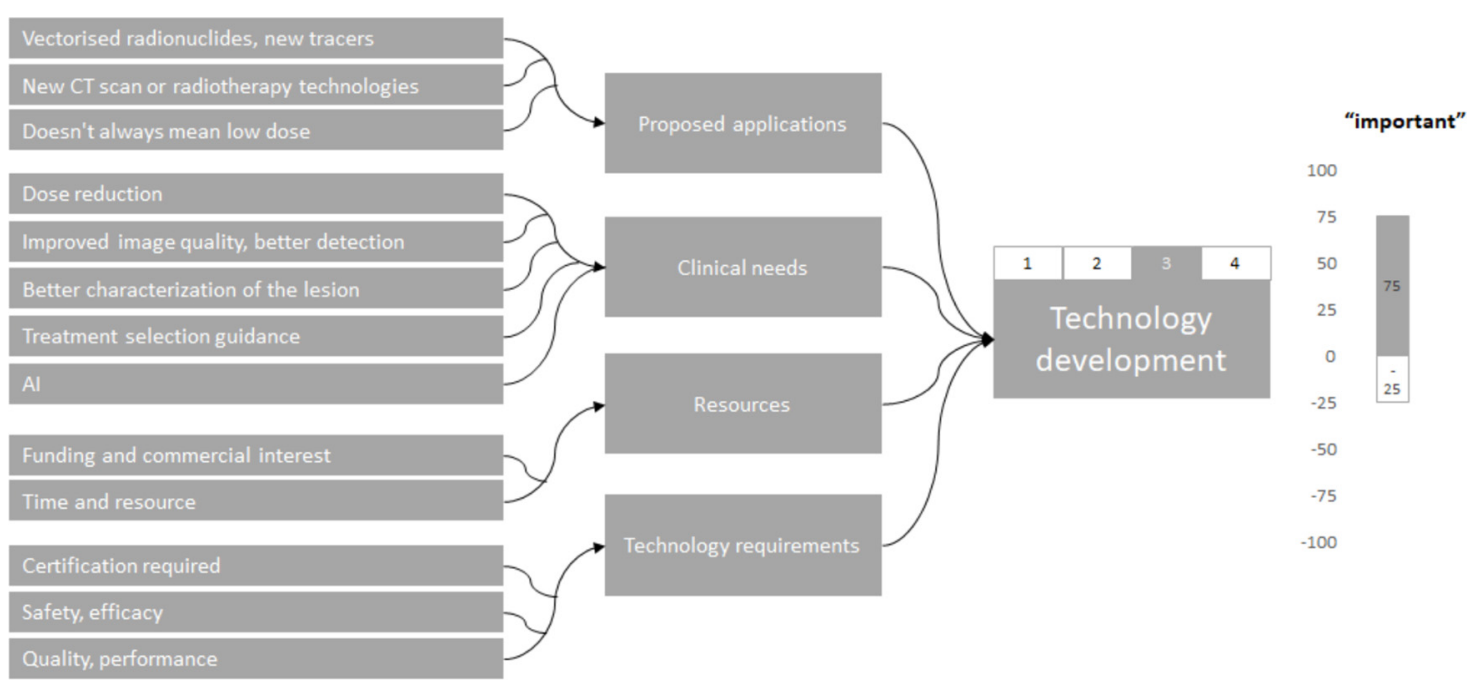

Fig. 7. Dendrogram for the OTHER MEDIRAD PRIORITY “Technology development”. 


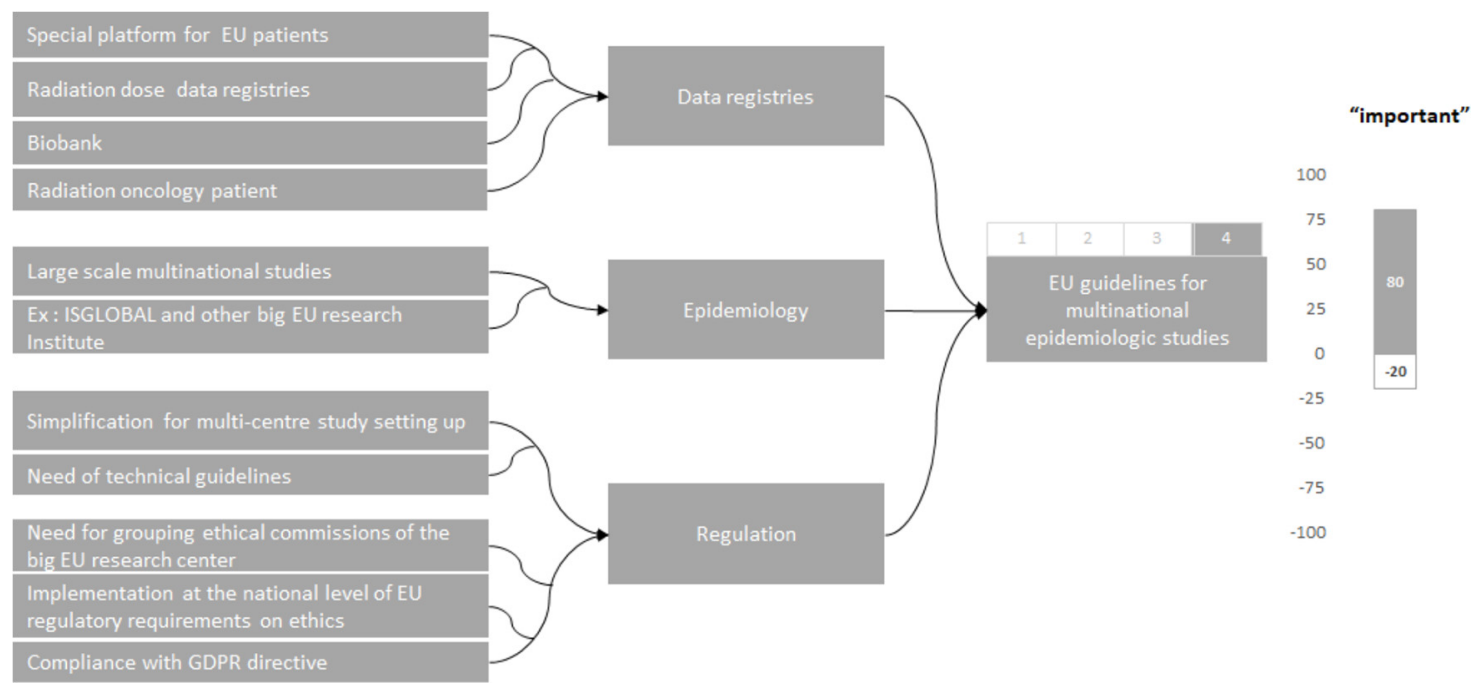

Fig. 8. Dendrogram for the OTHER MEDIRAD PRIORITY "Facilitating the development of large-scale multinational epidemiological studies by proposing guidelines to help European countries to implement at the national level European regulatory requirements on ethics (including compliance with GDPR directive)".

\section{Conclusion}

The purpose of exchanging views with the SF members on topics to be addressed by MEDIRAD recommendations was successfully achieved by the exploratory questionnaire. Eighty-five responses to the questionnaire were received, out of the 86 confirmed SF members. The Forum provides an uneven representation of European countries and stakeholder categories concerned by MEDIRAD research. Due to significant variation of interest among stakeholder groups targeted during the SF nomination process, those members who responded can be considered representative of the SF as a whole. The SF member's opinions on the various technical topics of the exploratory questionnaire were expressed through quantitative and qualitative methods. This analysis leads to numerical values and free text of comments, resulting in the identification of 11 coherent technical topics for possible MEDIRAD recommendation contents. This reflects a high level of consensus on their relevance, importance, and feasibility, towards the improvement of radiation protection of patients and medical professionals.

The answers to the questionnaire provided in detail clear evidence of support for further work in different areas towards (i) the development of standardised patient data repositories; (ii) further optimisation of diagnostic and therapeutic protocols; and more generally, (iii) on radiation protection issues in the medical context and the education of professionals on these topics in line with the literature (Hoeschen, 2018); and (iv) for research, the scientific development and implementation of new technologies. Fourteen additional topics showed a less significant degree of consensus. This does not necessarily mean that they are less important. However, it does imply that progress on these themes will have to take care of the need to improve stakeholder support, an essential condition to reach out successfully to communities of practice. The richness of exchanges among the SF members should encourage
MEDIRAD, and more generally, research partners funded by EURATOM in the field of medical radiation protection, to pursue broad multisectoral cooperation among academics, authorities, industry, users, and patients, in order to maximise the operational impact of scientific development in terms of protection of patients and medical personnel. Ionising radiation technologies constitute a major resource for medical diagnostics and therapies. In recent years, significant progress has been observed in this field, leading to improved imaging and therapeutic protocols and new possibilities to optimise radiation exposure, not only for patients but also for medical professionals. Further progress is bound to continue through technological innovation, good professional practice, and more individualised patient care.

This progress is based on continually accumulating scientific knowledge that can serve to address several public health challenges: (i) The rise in the number of medical examinations using ionising radiation, such as CT scans, has led to an increased collective dose for patients at the European level (European Union, 2018), with a potentially heightened risk for patients undergoing repeated procedures, especially for young patients. These risks are currently not well quantified. (ii) Latent risks to healthy tissues resulting from radiotherapy, interventional radiology and some nuclear medicine protocols have significantly been reduced with modern technologies. However, they remain an important issue to further consider as the rate of longterm survival in patients' increases (Bayart et al., 2019). (iii) Radiation protection for medical professionals with potentially high levels of exposure can still be further optimised.

\section{Acknowledgements}

This study is supported by the European Community's Horizon 2020 Programme, in the frame of the MEDIRAD-Implications of Medical Low Dose Radiation Exposure - A project for the period 2017-2021 (this project has received funding from the 
Euratom research and training programme 2014-2018 under grant agreement No. 755523).

\section{References}

Bayart E, Azria D, Balosso J, Benderitter M, Cohen-Jonathan Moyal E, Delpon G, Deutsch E, Dutreix M, Lacornerie T, Romeo PH, Marchesi V, Maingon P. 2019. RadioTransNet: le réseau national de radiothérapie oncologique préclinique. Cancer Radiother. 23 (6-7): 609-616.

Bernier M-O, Baysson H, Pearce MS, Moissonnier M, Cardis E, Hauptmann M, Struelens L, Dabin J, Johansen C, Journy N, Laurier D, Blettner M, Le Cornet L, Pokora R, Gradowska P, Meulepas JM, Kjaerheim K, Istad T, Olerud H, Sovik A. 2018. Cohort Profile: The EPI-CT study: a European pooled epidemiological study to quantify the risk of radiation-induced cancer from paediatric CT. Int. J. Epidemiol. 48(2): 379-381.

Borras JM, Lievens Y, Barton M, Corral J, Ferlay J, Bray F, Grau C. 2016. How many new cancer patients in Europe will require radiotherapy by 2025? An ESTRO-HERO analysis. Radiother. Oncol. 119(1): 5-11.

Bundesamt für Strahlenschutz (BfS-Dose Datamed 2). [online] Federal Office for Radiation Protection. Available from https:// www.bfs.de/EN/bfs/we/international/dose-datamed-2.html.

Euratom. 2013. European Council Directive 2013/59/Euratom of 5 December 2013 laying down basic safety standards for protection against the dangers arising from exposure to ionising radiation, and repealing Directives 89/618/Euratom, 90/641/Euratom, 96/29/Euratom, 97/43/Euratom and 2003/122/Euratom. Document 02013L0059-20140117 [online] Available from http://data.euro pa.eu/eli/dir/2013/59/2014-01-17 [Accessed 24 Feb. 2021].

European Association of Nuclear Medicine (EANM); European Federation of Organizations for Medical Physics (EFOMP); European Federation of Radiographer Societies (EFRS); European Society of Radiology (ESR); European Society for Radiotherapy and Oncology (ESTRO). 2017. Common strategic research agenda for radiation protection in medicine. Insights Imaging 8(2): 183-197.

European Commission (EC-Eurostat). Healthcare Resource Statistics-Technical Resources and Medical Technology-Statistics Explained. [online] ec.europa.eu. Available from https://ec. europa.eu/eurostat/statistics-explained/index.php/Healthcare_re source_statistics_-_technical_resources_and_medical_technol ogy. ISSN $2443-\overline{8} 2 \overline{1}$.

European Union. 2018. Radiation Protection $N^{\circ} 185$. European Guidelines on Diagnostic Reference Levels for Paediatric
Imaging. [online] Luxembourg: Publications Office of the European Union. Available from http://www.eurosafeimaging. org/wp/wp-content/uploads/2018/09/rp_185.pdf.

Francolini G, Desideri I, Stocchi G, Salvestrini V, Ciccone LP, Garlatti P, Loi M, Livi L. 2020. Artificial Intelligence in radiotherapy: state of the art and future directions. Med. Oncol. 37(6).

Gifi A. 1990. Nonlinear multivariate analysis. Chichester, New York: Wiley.

Hoeschen C. 2018. EURAMED's vision on medical radiation protection (research). Ann. ICRP 47(3-4): 152-158.

Hosny A, Parmar C, Quackenbush J, Schwartz LH, Aerts HJWL. 2018. Artificial intelligence in radiology. Nat. Rev. Cancer 18(8): 500-510. Available from https://www.ncbi.nlm.nih.gov/pmc/ articles/PMC6268174/.

ICRP Publication 135. 2017. Diagnostic reference levels in medical imaging. Ann. ICRP 46(1).

International Atomic Energy Agency. 2014. Radiation protection and safety of radiation sources: International basic safety standards. GSR Part 3. Vienna: IAEA.

International Atomic Energy Agency. 2018. Radiation protection and safety in medical uses of ionizing radiation: Specific safety guide. Vienna: IAEA.

Kaufman L, Rousueeuw PJ. 1990. Finding groups in data: An introduction to cluster analysis. John Wiley \& Sons.

Masjedi H, Zare MH, Keshavarz Siahpoush N, Razavi-Ratki SK, Alavi F, Shabani M. 2019. European trends in radiology: investigating factors affecting the number of examinations and the effective dose. La radiologia medica 125(3): 296-305.

Meskens G. 2020 Ethical considerations on stakeholder engagement in radiological risk governance. Radioprotection 55(HS2): S259-S262.

Rosenblatt E, Izewska J, Anacak Y, Pynda Y, Scalliet P, Boniol M, Autier P. 2013. Radiotherapy capacity in European countries: An analysis of the Directory of Radiotherapy Centres (DIRAC) database. Lancet Oncol. 14(2): e79-e86.

Skinner R, Wallace WHB, Levitt GA. 2006. Long-term follow-up of people who have survived cancer during childhood. Lancet Oncol. 7(6): 489-498.

Tsapaki V. 2020. Radiation dose optimization in diagnostic and interventional radiology: Current issues and future perspectives. Phys. Med. 79(79): 16-21.

Turcanu C, Van Oudheusden M, Abelshausen B, Schieber C, Schneider T, Zeleznik N, Geysmans R, Duranova T, Perko T, Pölzl-Viol C. 2020. Stakeholder engagement in radiological protection: Developing theory, practice and guidelines. Radioprotection 55(HS2): S211-S218.

Cite this article as: Benderitter M, Herrera Reyes E, Benadjaoud MA, Vanhavere F, Impens N, Mayerhofer-Sebera U, Hierath M, Jourdain JR, Frija G, Repussard J. 2021. MEDIRAD formulation of science-based recommendations for medical radiation protection: a stakeholder forum survey. Radioprotection 56(4): 275-285 